\title{
Kein Effekt auf die Prognose
}

Fragestellung: Inwiefern beeinflussen verschiedene Hämoglobinschwellenwerte sowie die Gabe von Erythropoetin (EPO) die neurologische Genesung nach Schädel-Hirn-Trauma (SHT)?

Hintergrund: Anämie stellt bei Patienten mit SHT eine mögliche Ursache für sekundäre neurologische Schäden dar, die das Outcome zusätzlich verschlechtern können. Nach vielversprechenden Ergebnissen in tierexperimentellen Arbeiten und kleineren Fallserien wurde erstmals in einer großen klinischen Studie untersucht, inwiefern sich die Behandlung der Anämie mit EPO und ein liberales Transfusionskonzept auf das Outcome auswirken.

Patienten und Methodik: Zwischen 2006 und 2012 wurden 200 neurochirurgische Intensivpatienten in den ersten sechs Stunden nach gedecktem SHT in die Studie eingeschlossen. Die Patienten waren nicht in der Lage, Anweisungen zu befolgen. Mittels eines faktoriellen Ver-

Robertson CS, Hannay H, Yamal J, et al. Effect of erythropoietin and transfusion threshold on neurological recovery after traumatic brain Injury: A randomized clinical trial. JAMA 2014; 312: 36-47 suchsplans $(2 \times 2)$ wurden die Gabe von Erythropoetin vs. Placebo sowie unterschiedliche Hämoglobinschwellenwerte (Transfusion von Erythrozytenkonzentraten entweder ab $7 \mathrm{~g} / \mathrm{dl}$ oder ab $10 \mathrm{~g} /$ dl) verglichen. Primärer End- punkt war der klinische Status gemessen an der Glasgow Outcome Scale nach sechs Monaten. Ferner wurden die Komplikationen obiger Interventionen erfasst. Intravenöses EPO (500 IU/ kg pro Gabe) oder Placebo wurden initial an den ersten drei Tagen täglich und danach wöchentlich für zwei weitere Wochen $(n=74)$ verabreicht, später wurde die Gabe am zweiten und dritten Tag ausgelassen $(n=126)$; diese nachträgliche Aufteilung der EPO-Patienten in verschiedene Dosierungsschemata entstand aufgrund von Sicherheitsbedenken der FDA. Bei 99 Patienten wurde der Hämoglobinschwellenwert bei $7 \mathrm{~g} / \mathrm{dl}$ und bei den restlichen 101 Patienten bei $10 \mathrm{~g} / \mathrm{dl}$ festgesetzt.

Ergebnisse: Keine der beiden EPO-Gruppen war gegenüber der Placebogruppe $(38,2 \%)$ überlegen. Patienten mit einem HbSchwellenwert von $7 \mathrm{~g} / \mathrm{dl}$ erreichten in $42,5 \%$ (vs. 33,0\% bei $10 \mathrm{~g} / \mathrm{dl}$ ) ein günstiges Outcome nach sechs Monaten, was ebenfalls nicht signifikant war. Allerdings wies das letztgenannte Therapiekonzept eine höhere thrombembolische Ereignisrate auf (Hb 10 g/dl: 21,8\% vs. Hb 7 g/dl: 8,1\%, p = 0,009).

Schlussfolgerungen: Bei Patienten mit geschlossenem SHT konnten weder die EPO-Gabe noch ein liberaleres Transfusionskonzept mit Aufrechterhalt der Hämoglobinwerte über $10 \mathrm{~g} / \mathrm{dl}$ das neurologische Outcome verbessern. Letztgenanntes Therapiekonzept zeigte zudem eine erhöhte Komplikationsrate.

\section{- Kommentar von Stefan Gerner und Hagen Huttner, Erlangen}

\section{Die Reihe der Negativstudien beim Menschen wurde fortgesetzt}

Eine Anämie ist immer wieder bei akuten neurologischen $\mathrm{Er}$ krankungen als möglicher Parameter, der das Outcome beeinflussen könnte, diskutiert worden. Dies basierte vorrangig auf kleineren Untersuchungen und tierexperimentellen Daten, die suggeriert hatten, dass die Aufrechterhaltung physiologischer Hämoglobinwerte die Sauerstoffversorgung des Gehirns in seiner Regenerationsphase verbessern könnte. Neben der Transfusion von Erythrozytenkonzentraten steht auch die Gabe von EPO zur Verfügung, dem zusätzlich eine antiapoptotische und antiinflammatorische Wirkung zugesprochen wird. Nachdem bereits 1999 im Rahmen der TRICC-Studie an einem großen Intensivkollektiv ein restriktiveres Transfusionsregime einem liberaleren beinahe überlegen war, und ferner die Gabe von EPO sich bei Schlaganfallpatienten als nicht wirkungsvoll erwiesen hatte, reiht sich diese neurochirurgische SHT-Studie in die bisherigen Negativbeobachtungen am Menschen ein.

Allerdings unterminiert eine Reihe gravierender Mängel die Aussagekraft dieser Studie. So erscheint es mehr als unangebracht, eine klinische Effektgröße von $20 \%$ durch die Gabe von EPO anzunehmen und darauf basierend die nötige Patientenzahl durch eine Poweranalyse zu kalkulieren; entsprechend kleine Patientenzahlen, die auch noch in vier Gruppen unterteilt wurden, limitierten de facto die Schlussfolgerungen sämtlicher
Ergebnisse. Zudem wurde aufgrund von Sicherheitsbedenken der FDA die Studie für zirka ein Jahr ausgesetzt und die Dosierung von EPO unter die Werte gesenkt, die sich in tierexperimentellen Ansätzen als wirkungsvoll herausgestellt hatten; dies führte auch zu einer nachträglichen Änderung des Designs weg von einer "Superiority-“" hin zu einer "Futility"-Analyse. Auch wurde nicht zwischen einer Anämie, die bereits bei Aufnahme vorlag, und einer Anämie, die sich während des stationären Aufenthaltes entwickelte, unterschieden.

Die Frage, ob eher die Anämie selbst oder ihre Behandlung zur sekundären Schädigung bei SHT-Patienten beiträgt, kann durch diese Studie nicht geklärt werden.

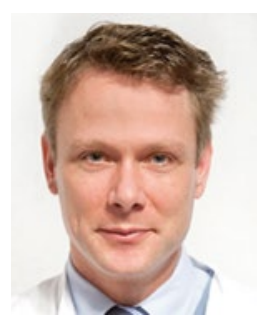

PD Dr. med. Hagen B. Huttner, Erlangen

Universitätsklinik für Neurologie, Erlangen E-Mail:hagen.huttner@uk-erlangen.de 